\title{
Density matrix functional theory that includes pairing correlations
}

\author{
S. Krewald, ${ }^{1}$ V. B. Soubbotin, ${ }^{2}$ V. I. Tselyaev, ${ }^{2}$ and X. Viñas ${ }^{3}$ \\ ${ }^{1}$ Institut für Kernphysik, Forschungszentrum Jülich, D-52425 Jülich, Germany \\ ${ }^{2}$ Nuclear Physics Department, V.A. Fock Institute of Physics, St. Petersburg State University, RU-198504, St. Petersburg, Russia \\ ${ }^{3}$ Departament d'Estructura i Constituents de la Matèria, Facultat de Física, Universitat de Barcelona, \\ Diagonal 645, E-08028 Barcelona, Spain
}

(Received 20 December 2004; revised manuscript received 11 August 2006; published 13 December 2006)

\begin{abstract}
The extension of density functional theory (DFT) to include pairing correlations without formal violation of the particle-number conservation condition is described. This version of the theory can be considered as a foundation of the application of existing DFT plus pairing approaches to atoms, molecules, ultracooled and magnetically trapped atomic Fermi gases, and atomic nuclei where the number of particles is conserved exactly. The connection with Hartree-Fock-Bogoliubov (HFB) theory is discussed, and the method of quasilocal reduction of the nonlocal theory is also described. This quasilocal reduction allows equations of motion to be obtained which are much simpler for numerical solution than the equations corresponding to the nonlocal case. Our theory is applied to the study of some even Sn isotopes, and the results are compared with those obtained in the standard HFB theory and with the experimental ones.
\end{abstract}

DOI: 10.1103/PhysRevC.74.064310

PACS number(s): 21.60.Jz, 27.60.+j, 31.15.Ew, 74.20.-z

\section{INTRODUCTION}

Although both the theory of superconductivity and density functional theory (DFT) have a long history, the models which take into account pairing correlations within the DFT framework have only appeared fairly recently. The initial version of DFT developed in the early papers of Hohenberg, Kohn, and Sham [1,2] did not include the pairing correlations explicitly. The first generalization of DFT in this direction was developed in Ref. [3] for superconductors (see also Refs. [4,5] where modifications of this approach were developed). However, the essential feature of this theory is the nonconservation of the number of particles in the superconducting Fermi system. Consequently, the application of DFT for superconductors to atoms, molecules, ultracooled and magnetically trapped atomic Fermi gases, and atomic nuclei, where the pairing correlations may be important but the number of particles is exactly conserved, requires an additional foundation. In fact, the same question emerges in connection with DFT plus pairing approaches based, for example, on the theory of finite Fermi systems by Migdal (see [6] and references therein) or on the local density approximation (Ref. [7]). Thus, the first goal of the present paper is a rigorous formulation of the extended version of DFT taking into account the pairing correlations under the condition of particle-number conservation.

The second aim is to extend DFT to the case of a functional dependence on the total nonlocal single-particle density matrix (DM). Such an extension is especially important in applications to nuclei because it allows the dependence of the energy functional on the densities of the kinetic energy and the spin to be introduced in a natural way. This kind of dependence leads to the appearance of a radial-dependent effective mass and a spin-orbit potential, which are essential components of the nuclear structure models. Let us note that the original version of DFT can be classed as a local theory because in the papers $[1,2]$ the energy functional only depends on the local particle density. One possible way that leads to a nonlocal extension of the DFT was considered in Ref. [8]. However, that method, which can be referred to as a straightforward extension, faces serious difficulties related to equations of motion and their physical interpretation. Another method was recently developed in Ref. [9] to extend the DFT by considering an energy functional which depends on a Slater-determinant DM. That approach leads to the quasilocal density functional theory and avoids the difficulties arising from the method described in [8]. However, in general, the resulting DM is not the exact DM because its diagonal part is the only quantity which coincides with the exact local particle density of the interacting fermion system.

In the present paper, we will show that including pairing within the framework of the extended DFT is sufficient to obtain the exact total nonlocal DM on the one hand and to avoid the difficulties encountered in the equations of motion of Ref. [8] on the other. Although we develop our formalism in the particular case of the atomic nucleus, the main results and conclusions can also be applied to any Fermi system with a fixed number of particles. The paper is organized as follows. In Sec. II, the extended density matrix formalism is revised. In Sec. III, the DFT is extended to include pairing correlations. In Sec. IV, the reduction to an extended quasilocal density functional theory is performed. In Sec. V, the quasilocal version of our approach is applied to describe the ground-state energies and the average gaps of some tin isotopes near the $\beta$-stability valley. In our calculation, the pairing correlations are treated at the BCS level, and the results are compared with the full Hartree-Fock-Bogoliubov and with the experimental ones. Finally, conclusions are given in the last section. In the 
Appendices, some mathematical details and comments are presented.

\section{EXTENDED DENSITY MATRIX FORMALISM}

Even though the formalism of the density matrix extended to include pairing correlations in the ground state is well known (see, e.g., Refs. [10,11]), we shall provide some basic definitions which are necessary for further analysis. Let $\Psi$ be some arbitrary antisymmetrized many-fermion wave function. In the general case, $\Psi$ is assumed to be normalized, but it is not assumed to be an eigenfunction of the particle-number operator. Thus, the normal $(\rho)$ and the anomalous $(\kappa)$ density matrices in the state $\Psi$ are defined through the following expectation values:

$$
\begin{aligned}
\rho\left(x, x^{\prime}\right) & =\left\langle\Psi\left|a^{\dagger}\left(x^{\prime}\right) a(x)\right| \Psi\right\rangle, \\
\kappa\left(x, x^{\prime}\right) & =\left\langle\Psi\left|a\left(x^{\prime}\right) a(x)\right| \Psi\right\rangle, \\
\kappa^{*}\left(x, x^{\prime}\right) & =\left\langle\Psi\left|a^{\dagger}(x) a^{\dagger}\left(x^{\prime}\right)\right| \Psi\right\rangle,
\end{aligned}
$$

where $a^{\dagger}(x)$ and $a(x)$ are creation and annihilation operators of particles in the coordinate representation of the usual single-particle space. In the case of atomic nuclei, symbol $x=\{r, \sigma, q\}$ includes the spatial coordinate $r$ and the spin projection $\sigma$ variables as well as the index $q=n, p$ which indicates the nucleon type (neutrons and protons).

It is important to note that for the Fermi systems considered here, the ground state (GS) is described by a wave function $\Psi=\Psi_{\mathrm{GS}}$ with a fixed number of particles. Therefore, in the GS the anomalous DM vanishes:

$$
\kappa_{\mathrm{GS}}\left(x, x^{\prime}\right)=0 .
$$

However, in many physical problems, one can construct auxiliary quantities which have the sense of an anomalous DM but, nevertheless, take nonzero values in the GS even if Eq. (3) is fulfilled.

Let us suppose that the DM $\rho$ defined by Eq. (1) is given for some wave function $\Psi$ (in particular, it may be the exact GS wave function $\Psi_{\mathrm{GS}}$ ). If $\Psi$ is time-reversal invariant, we can introduce the canonical basis (CB) $\left\{\phi_{\lambda}(x)\right\}$, where the singleparticle multi-index $\lambda$ contains the sign of the spin projection $s$ and the set of the remaining quantum numbers $\{c\}(\lambda=\{c, s\})$, so that the following expansion is fulfilled:

$$
\rho\left(x, x^{\prime}\right)=\sum_{c, s} v_{c}^{2} \phi_{c, s}(x) \phi_{c, s}^{*}\left(x^{\prime}\right), \quad 0 \leqslant v_{c} \leqslant 1 .
$$

In the case of real Fermi systems, this DM is not idempotent (i.e., $\rho^{2} \neq \rho$ ). This is because all or almost all of the eigenvalues of $\rho$, defined by the equation $\int d x^{\prime} \rho\left(x, x^{\prime}\right) \phi_{c, s}\left(x^{\prime}\right)=$ $v_{c}^{2} \phi_{c, s}(x)$ (hereinafter $\int d x$ means the space integral over $r$ and the sum over $\sigma$ and $q$ indices), lie in the interval $0<v_{c}^{2}<1$. If one uses, as done in the theory developed by Gilbert [8], an energy functional $E_{G}[\rho]$ that depends only on such a nonlocal, non-idempotent DM, then in the resulting equations of motion, all partially occupied natural spin orbitals [i.e., functions $\phi_{c, s}(x)$ for which $0<v_{c}^{2}<1$ ] are eigenfunctions of the single-particle pseudo-Hamiltonian $h_{G}\left(x, x^{\prime}\right)=\delta E_{G}[\rho] / \delta \rho\left(x^{\prime}, x\right)$ with the same eigenvalue (see Ref. [8] for details). This fact leads to difficulties in the physical interpretation and in the mathematical foundation of the theory developed by Gilbert. To avoid this problem, first of all, we shall define an extended density matrix (EDM) $\mathcal{R}$ which has to be idempotent $\left(\mathcal{R}^{2}=\mathcal{R}\right)$ and has to contain a given DM $\rho$ as a block. An EDM is a DM that is defined in a space that is double the size of the usual single-particle space.

Let $\left\{\psi_{\lambda ; \eta}(x ; \chi)\right\}$ be an arbitrary set of basis functions in this extended space spanned by the coordinates $\{x, \chi\}$, where $\chi= \pm 1$ and $\eta= \pm 1$ are additional indices introduced to denote the different components of the single-particle functions. The meaning of these indices will be specified in the following. [In particular, the index $\chi$ indicates the upper and lower components of the functions $\psi_{\lambda ; \eta}(x ; \chi)$ according to the notation in Ref. [11]]. The usual conditions of orthonormality and completeness are assumed to be satisfied, that is,

$$
\begin{aligned}
\sum_{\chi} \int d x \psi_{\lambda ; \eta}^{*}(x ; \chi) \psi_{\lambda^{\prime} ; \eta^{\prime}}(x ; \chi) & =\delta_{\eta, \eta^{\prime}} \delta_{\lambda, \lambda^{\prime}}, \\
\sum_{\lambda, \eta} \psi_{\lambda ; \eta}^{*}(x ; \chi) \psi_{\lambda ; \eta}\left(x^{\prime} ; \chi^{\prime}\right) & =\delta_{\chi, \chi^{\prime}} \delta\left(x, x^{\prime}\right),
\end{aligned}
$$

where $\delta\left(x, x^{\prime}\right)=\delta\left(\boldsymbol{r}-\boldsymbol{r}^{\prime}\right) \delta_{\sigma, \sigma^{\prime}} \delta_{q, q^{\prime}}$. In addition, we also assume that the functions $\psi_{\lambda ; \eta}(x ; \chi)$ satisfy the condition

$$
\psi_{\lambda ; \eta}(x ; \chi)=\psi_{\lambda ;-\eta}^{*}(x ;-\chi)
$$

From the conditions (5) and (6) it follows that the functions $\psi_{\lambda ; \eta}(x ; \chi)$ form a unitary matrix in the extended space defined previously. (Strictly speaking, they form a unitary operator. The use of the term matrix implies that the configuration space is discretized and restricted to a finite number of points.) If the condition (7) is also fulfilled, the Bloch-Messiah theorem (see Refs. [10,12]) can be applied to this matrix. In order to reformulate this theorem in the coordinate representation, let us first introduce a complete set of orthonormal functions $\left\{\tilde{\phi}_{\lambda}(x)\right\}$. Notice that these functions form a unitary matrix $D$ in the notation of Ref. [10] according to the rule $D_{i k}=\tilde{\phi}_{\lambda_{k}}\left(x_{i}\right)$. Second, let us divide the set of the single-particle indices $\lambda$ into three subsets: two sets of conjugate indices $p$ and $\bar{p}$ which represent "paired" states, and the set of indices $b$ corresponding to "blocked" states, i.e., $\{\lambda\}=\{p\} \cup\{\bar{p}\} \cup\{b\}$. Furthermore, let $\tilde{v}_{\lambda}$ and $\tilde{u}_{\lambda}$ be real non-negative numbers that satisfy the following conditions: $\tilde{u}_{\lambda}=\sqrt{1-\tilde{v}_{\lambda}^{2}}, \tilde{v}_{p}=\tilde{v}_{\bar{p}}, 0<\tilde{v}_{p}<1$, and $\tilde{v}_{b}^{2}=\tilde{v}_{b}$. According to the Bloch-Messiah theorem, the functions $\psi_{\lambda ; \eta}(x ; \chi)$ can be represented in the form

$$
\begin{aligned}
& \psi_{\lambda ;+}(x ; \chi)=\sum_{\lambda^{\prime}} C_{\lambda^{\prime} \lambda} \breve{\psi}_{\lambda^{\prime} ;+}(x ; \chi), \\
& \psi_{\lambda ;-}(x ; \chi)=\sum_{\lambda^{\prime}} C_{\lambda^{\prime} \lambda}^{*} \breve{\psi}_{\lambda^{\prime} ;-}(x ; \chi),
\end{aligned}
$$


where $C_{\lambda^{\prime} \lambda}$ is a unitary matrix, and the functions $\check{\psi}_{\lambda ; \eta}(x ; \chi)$ have the form

$$
\left.\begin{array}{lll}
\check{\psi}_{p ;+}(x ;+)=\tilde{u}_{p} \tilde{\phi}_{p}(x), & \check{\psi}_{\bar{p} ;+}(x ;+)=\tilde{u}_{p} \tilde{\phi}_{\bar{p}}(x), & \check{\psi}_{b ;+}(x ;+)=\tilde{u}_{b} \tilde{\phi}_{b}(x), \\
\check{\psi}_{p ;+}(x ;-)=-\tilde{v}_{p} \tilde{\phi}_{\bar{p}}^{*}(x), & \check{\psi}_{\bar{p} ;+}(x ;-)=\tilde{v}_{p} \tilde{\phi}_{p}^{*}(x), & \check{\psi}_{b ;+}(x ;-)=\tilde{v}_{b} \tilde{\phi}_{b}^{*}(x), \\
\check{\psi}_{p ;-}(x ;+)=-\tilde{v}_{p} \tilde{\phi}_{\bar{p}}(x), & \check{\psi}_{\bar{p} ;-}(x ;+)=\tilde{v}_{p} \tilde{\phi}_{p}(x), & \check{\psi}_{b ;-}(x ;+)=\tilde{v}_{b} \tilde{\phi}_{b}(x), \\
\check{\psi}_{p ;-}(x ;-)=\tilde{u}_{p} \tilde{\phi}_{p}^{*}(x), & \check{\psi}_{\bar{p} ;-}(x ;-)=\tilde{u}_{p} \tilde{\phi}_{\bar{p}}^{*}(x), & \check{\psi}_{b ;-}(x ;-)=\tilde{u}_{b} \tilde{\phi}_{b}^{*}(x) .
\end{array}\right\}
$$

Let us now define the EDM $\mathcal{R}$ in terms of the arbitrary set of functions $\left\{\psi_{\lambda ; \eta}(x ; \chi)\right\}$ introduced above by the formula

$$
\mathcal{R}\left(x, \chi ; x^{\prime}, \chi^{\prime}\right)=\sum_{\lambda} \psi_{\lambda ;-}(x ; \chi) \psi_{\lambda ;-}^{*}\left(x^{\prime} ; \chi^{\prime}\right)
$$

Using Eqs. (5)-(7), it can be easily shown that the following equalities are fulfilled:

$$
\begin{aligned}
\mathcal{R}^{2} & =\mathcal{R}, \quad \mathcal{R}^{\dagger}=\mathcal{R}, \\
\mathcal{R}\left(x, \chi ; x^{\prime}, \chi^{\prime}\right) & =\delta_{\chi, \chi^{\prime}} \delta\left(x, x^{\prime}\right)-\mathcal{R}\left(x^{\prime},-\chi^{\prime} ; x,-\chi\right) .
\end{aligned}
$$

Let us introduce notation for the blocks of the EDM taking into account the properties (11)-(12):

$$
\left.\begin{array}{ll}
\mathcal{R}\left(x,+; x^{\prime},+\right)=\tilde{\rho}\left(x, x^{\prime}\right), & \mathcal{R}\left(x,+; x^{\prime},-\right)=\tilde{\kappa}\left(x, x^{\prime}\right), \\
\mathcal{R}\left(x,-; x^{\prime},+\right)=-\tilde{\kappa}^{*}\left(x, x^{\prime}\right), & \mathcal{R}\left(x,-; x^{\prime},-\right)=\delta\left(x, x^{\prime}\right)-\tilde{\rho}^{*}\left(x, x^{\prime}\right),
\end{array}\right\}
$$

or in the matrix representation

$$
\mathcal{R}=\left(\begin{array}{ll}
\tilde{\rho} & \tilde{\kappa} \\
-\tilde{\kappa}^{*} & 1-\tilde{\rho}^{*}
\end{array}\right) .
$$

As will be clear in the following [see Eqs. (21)-(22) below], the blocks $\tilde{\rho}$ and $\tilde{\kappa}$ play the role of the normal and anomalous density matrices in some (quasiparticle vacuum) state.

Substituting Eqs. (8) and (9) into Eq. (10), we obtain

$$
\tilde{\rho}\left(x, x^{\prime}\right)=\sum_{\lambda} \tilde{v}_{\lambda}^{2} \tilde{\phi}_{\lambda}(x) \tilde{\phi}_{\lambda}^{*}\left(x^{\prime}\right),
$$

where the sum over $\lambda$ implies the sum over the three sets of indices $p, \bar{p}$, and $b$. Thus, the functions $\tilde{\phi}_{\lambda}(x)$ form the CB in which $\tilde{\rho}\left(x, x^{\prime}\right)$ is diagonal. Thus, in the following we shall refer to Eq. (9) as the CB representation of the functions $\psi_{\lambda ; \eta}(x ; \chi)$.

Comparing Eqs. (4) and (15), we see that the expansion (4) is a particular case of (15). Indeed, setting the number of blocked occupied states in (15) to be even or to be equal to zero, one can choose $\tilde{v}_{\lambda}=v_{\lambda}, \tilde{\phi}_{\lambda}(x)=\phi_{\lambda}(x)$, which leads to the coincidence of the right-hand sides of Eqs. (4) and (15). So, there is a variety of sets of the functions $\psi_{\lambda ; \eta}(x ; \chi)$, which enter the definition (10) and differ by an arbitrary unitary transformation of the type Eq. (8), such that the following equalities are fulfilled:

$$
\mathcal{R}\left(x,+; x^{\prime},+\right)=\tilde{\rho}\left(x, x^{\prime}\right)=\rho\left(x, x^{\prime}\right) .
$$

Because Eq. (4) is fulfilled for any physically meaningful $\mathrm{DM} \rho$, we have actually proved that for an arbitrary nonlocal
DM $\rho\left(x, x^{\prime}\right)$, corresponding to some interacting time-reversal invariant fermion system, we can construct an EDM $\mathcal{R}$ which satisfies the conditions (11)-(12) and which is related to the DM $\rho$ by the formula (16).

It is important to note that if the normal $\mathrm{DM} \rho$ is produced by some wave function $\Psi$ according to Eq. (1), the anomalous DM $\kappa$ produced by the same wave function $\Psi$ according to Eqs. (2) does not coincide, in general, with the quantity $\tilde{\kappa}$ defined as a block of the EDM by Eqs. (13), even if Eqs. (16) are fulfilled. In particular, if $\Psi=\Psi_{\mathrm{GS}}$ then, as was mentioned above, $\kappa=\kappa_{\mathrm{GS}}=0$; while for the interacting system, $\tilde{\kappa} \neq 0$. On the other hand, if $\Psi$ is a quasiparticle-vacuum wave function (see below), then the equality $\kappa=\tilde{\kappa}$ is fulfilled.

To reproduce an arbitrarily given DM $\rho\left(x, x^{\prime}\right)$ as a block of the EDM $\mathcal{R}$, we started from an arbitrary complete set of basis functions $\left\{\psi_{\lambda ; \eta}(x ; \chi)\right\}$ which satisfy the conditions (5)-(7). It is useful to carry out this step in a different way, looking at this problem from a more traditional point of view. To this end, let us introduce the creation and annihilation operators of the quasiparticles $\alpha_{\lambda}^{\dagger}$ and $\alpha_{\lambda}$ through the equation

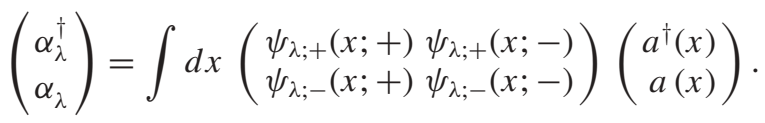

With this definition, the functions $\psi_{\lambda ; \eta}(x ; \chi)$ form the matrix of the Bogoliubov transformation. The properties (5)-(7) then simply follow from the requirement of the unitarity of this transformation and from the fact that $\alpha_{\lambda}^{\dagger}$ and $\alpha_{\lambda}$ are a Hermitian 
conjugate pair of operators. The inverse relation, which follows from (6) and (7), reads

$$
\left(\begin{array}{c}
a^{\dagger}(x) \\
a(x)
\end{array}\right)=\sum_{\lambda}\left(\begin{array}{c}
\psi_{\lambda ;-}(x ;-) \psi_{\lambda ;+}(x ;-) \\
\psi_{\lambda ;-}(x ;+) \psi_{\lambda ;+}(x ;+)
\end{array}\right)\left(\begin{array}{c}
\alpha_{\lambda}^{\dagger} \\
\alpha_{\lambda}
\end{array}\right) .
$$

Let us define the quasiparticle-vacuum wave function $\tilde{\Psi}$ by the ordinary condition

$$
\alpha_{\lambda}|\tilde{\Psi}\rangle=0, \quad \forall \lambda
$$

This definition is unique, and the wave function $\tilde{\Psi}$ defined by the condition (19) is invariant under the $C$ transformation (8). Thus, one can find the explicit general form of $\tilde{\Psi}$ by using the CB representation (9). This result is well known (see, e.g., Ref. [10]) and reads

$$
|\tilde{\Psi}\rangle=\prod_{b}\left(\tilde{u}_{b}+\tilde{v}_{b} a_{b}^{\dagger}\right) \prod_{p}\left(\tilde{u}_{p}+\tilde{v}_{p} a_{p}^{\dagger} a_{\bar{p}}^{\dagger}\right)|0\rangle,
$$

where $|0\rangle$ is the particle-vacuum wave function $a_{\lambda}^{\dagger}=$ $\int d x \tilde{\phi}_{\lambda}(x) a^{\dagger}(x)$. If, furthermore, we formally define the EDM $\mathcal{R}$ through Eqs. (13) with

$$
\begin{gathered}
\tilde{\rho}\left(x, x^{\prime}\right)=\left\langle\tilde{\Psi}\left|a^{\dagger}\left(x^{\prime}\right) a(x)\right| \tilde{\Psi}\right\rangle, \\
\tilde{\kappa}\left(x, x^{\prime}\right)=\left\langle\tilde{\Psi}\left|a\left(x^{\prime}\right) a(x)\right| \tilde{\Psi}\right\rangle, \\
\tilde{\kappa}^{*}\left(x, x^{\prime}\right)=\left\langle\tilde{\Psi}\left|a^{\dagger}(x) a^{\dagger}\left(x^{\prime}\right)\right| \tilde{\Psi}\right\rangle,
\end{gathered}
$$

then it is easy to show using Eqs. (18) and (19) that this EDM is expressed in terms of the functions $\psi_{\lambda ; \eta}(x ; \chi)$ by Eq. (10) and consequently satisfies Eqs. (11)-(12). In other words, Eqs. (13), (21), (22) set a mapping of $\tilde{\Psi}$ onto the EDM $\mathcal{R}$ satisfying Eqs. (10)-(12). In the following, the many-to-one mappings of the many-fermion wave functions (e.g., $\tilde{\Psi})$ to the density matrices (e.g., $\tilde{\rho}$ or $\mathcal{R}$ ), defined by equations of the type (13), (21), and (22), will be denoted as $\tilde{\Psi} \rightarrow \tilde{\rho}$ or $\tilde{\Psi} \rightarrow \mathcal{R}$. Because the matrix elements of the Bogoliubov transformation $\psi_{\lambda ; \eta}(x ; \chi)$ in the above derivation are constrained only by conditions (5)-(7), the existence of the pointed mapping $\tilde{\Psi} \rightarrow \mathcal{R}$ is true, in particular, if $\psi_{\lambda ; \eta}(x ; \chi)$ are chosen in such a way that Eqs. (16) are fulfilled for some arbitrarily given $\mathrm{DM} \rho$ (which is always possible, as was proved above). We then also have the mapping $\tilde{\Psi} \rightarrow \mathcal{R} \rightarrow \rho$. Consequently, it can be argued that for an arbitrary nonlocal DM $\rho\left(x, x^{\prime}\right)$, corresponding to some interacting time-reversal invariant fermion system, including the case for which the number of particles is exactly conserved, there is a quasiparticle-vacuum wave function $\tilde{\Psi}$ such that $\tilde{\Psi} \rightarrow \rho$. The explicit form of $\tilde{\Psi}$ is given by Eq. (20), in which $\tilde{v}_{\lambda}^{2}$ and $\tilde{\phi}_{\lambda}(x)$ are the eigenvalues $v_{\lambda}^{2}$ and the eigenfunctions $\phi_{\lambda}(x)$ of the given DM $\rho$. The opposite is also true: any quasiparticle-vacuum wave function that satisfies the condition $\tilde{\Psi} \rightarrow \rho$ has the general explicit form (20) with $\tilde{u}_{\lambda}=u_{\lambda}, \tilde{v}_{\lambda}=v_{\lambda}$, and $\tilde{\phi}_{\lambda}(x)=\phi_{\lambda}(x)$.

These statements can be considered as a generalization of the Lieb theorem [13] proved for the local particle density and the Slater-determinant wave functions. It is remarkable that including pairing is sufficient not only to prove a more general statement but also to make the proof simpler. Moreover, the Lieb proof is based on the particular example of a Slater-determinant wave function which produces a given local particle density. However, in the Lieb theorem, the general explicit form of such a Slater-determinant wave function is not constructed. In contrast, in our case, we know the general explicit form (20) of the quasiparticle-vacuum wave function that satisfies the condition $\tilde{\Psi} \rightarrow \rho$.

\section{EXTENSION OF THE DFT}

Let $H$ be the nonrelativistic exact many-body Hamiltonian of an interacting fermion system. Let us define an auxiliary functional which depends only on the normal nonlocal DM $\rho$ :

$$
E[\rho]=\inf _{\Psi \rightarrow \rho}\langle\Psi|H| \Psi\rangle,
$$

where $\Psi$ are arbitrary normalized many-fermion wave functions, including those with a fixed number of particles. Following the method of Ref. [9], let us introduce an effective many-body Hamiltonian $\tilde{H}$ which generally does not coincide with $H$. Now we define

$$
\tilde{\mathcal{E}}\left[\rho, \tilde{\kappa}, \tilde{\kappa}^{*}\right]=\inf _{\tilde{\Psi} \rightarrow \rho, \tilde{\kappa}, \tilde{\kappa}^{*}}\langle\tilde{\Psi}|\tilde{H}| \tilde{\Psi}\rangle,
$$

where $\tilde{\Psi}$ are the quasiparticle-vacuum wave functions. Thanks to the existence of the mapping $\tilde{\Psi} \rightarrow \rho$ proved in the previous section, the functional $\tilde{\mathcal{E}}\left[\rho, \tilde{\kappa}, \tilde{\kappa}^{*}\right]$ can be defined for an arbitrary nonlocal DM $\rho\left(x, x^{\prime}\right)$ corresponding to some interacting time-reversal invariant fermion system with a fixed number of particles and for those matrices $\tilde{\kappa}, \tilde{\kappa}^{*}$ which are produced by the quasiparticle-vacuum wave functions according to Eqs. (22). Notice, however, that Eq. (24) implies that $\tilde{H}$ is not a completely arbitrary operator because it is constrained by some mathematical conditions. First, the energy functional $\tilde{\mathcal{E}}$ has to be well defined. This is not a trivial property (in spite of the existing mapping $\tilde{\Psi} \rightarrow \rho$ ) because, in the case of atomic nuclei, the expectation value of the exact many-body Hamiltonian $H$ obtained with the quasiparticle-vacuum wave function can diverge because of the short-range singularity of the bare nucleon-nucleon $(N N)$ forces. Thus, it is assumed that the effective Hamiltonian $\tilde{H}$ contains $N N$ forces whose matrix elements are well defined. Second, $\tilde{H}$ has to be chosen to ensure the minimal property of the total energy functional [see Eq. (26)) below and Ref. [9] for more details]. Using Eqs. (23) and (24) we can define, in analogy with Ref. [9], the residual correlation energy $E_{\mathrm{RC}}$

$$
E_{\mathrm{RC}}[\rho]=E[\rho]-\inf _{\tilde{\kappa}, \tilde{\kappa}^{*}} \tilde{\mathcal{E}}\left[\rho, \tilde{\kappa}, \tilde{\kappa}^{*}\right],
$$

and the total energy functional $\mathcal{E}$

$$
\mathcal{E}\left[\rho, \tilde{\kappa}, \tilde{\kappa}^{*}\right]=\tilde{\mathcal{E}}\left[\rho, \tilde{\kappa}, \tilde{\kappa}^{*}\right]+E_{\mathrm{RC}}[\rho] .
$$

The main property of the total functional $\mathcal{E}\left[\rho, \tilde{\kappa}, \tilde{\kappa}^{*}\right]$ is

$$
\begin{aligned}
\inf _{\rho, \tilde{\kappa}, \tilde{\kappa}^{*}} \mathcal{E}\left[\rho, \tilde{\kappa}, \tilde{\kappa}^{*}\right] & =\inf _{\rho}\left(\inf _{\tilde{\kappa}, \tilde{\kappa}^{*}} \tilde{\mathcal{E}}\left[\rho, \tilde{\kappa}, \tilde{\kappa}^{*}\right]+E_{\mathrm{RC}}[\rho]\right) \\
& =\inf _{\rho} E[\rho]=E_{\mathrm{GS}},
\end{aligned}
$$

where $E_{\mathrm{GS}}$ is the exact ground-state energy of the interacting system. If the infimum of the total functional (26) is the minimum (a usual assumption which has to be fulfilled by an appropriate choice of the effective Hamiltonian $\tilde{H}$ ), 
this minimum is achieved for the true nonlocal ground-state DM $\rho$ as follows from Eq. (23) and from the results of Sec. II.

It is advisable to pass from the variables $\rho, \tilde{\kappa}$, and $\tilde{\kappa}^{*}$ in the functional $\mathcal{E}$ to the components of the EDM $\mathcal{R}$ using Eqs. (13) for $\tilde{\kappa}$ and $\tilde{\kappa}^{*}$ and the relation

$$
\rho\left(x, x^{\prime}\right)=\frac{1}{2}\left[\delta\left(x^{\prime}, x\right)-\mathcal{R}\left(x^{\prime},-; x,-\right)+\mathcal{R}\left(x,+; x^{\prime},+\right)\right],
$$

which follows from Eqs. (13) at $\tilde{\rho}=\rho$. Taking into account these relations, we introduce the energy functional $\mathcal{E}_{\text {ext }}$ which depends on the EDM

$$
\mathcal{E}_{\text {ext }}[\mathcal{R}]=\mathcal{E}\left[\rho, \tilde{\kappa}, \tilde{\kappa}^{*}\right] .
$$

It is obvious from Eqs. (27) and (29) that $\inf _{\mathcal{R}} \mathcal{E}_{\text {ext }}[\mathcal{R}]=E_{\mathrm{GS}}$.

To establish the equations of motion of the theory, which in the following will be referred to as the extended density matrix functional theory (EDMFT), let us define the functional

$$
\begin{aligned}
F\left[\psi_{\lambda ;-}, \psi_{\lambda ;-}^{*}\right]= & \mathcal{E}_{\mathrm{ext}}[\mathcal{R}]+\frac{1}{2} \sum_{\lambda, \chi} E_{\lambda} \int d x\left|\psi_{\lambda ;-}(x ; \chi)\right|^{2} \\
& -\frac{1}{2} \int d x d x^{\prime} \mu\left(x, x^{\prime}\right)\left[\delta\left(x^{\prime}, x\right)\right. \\
& \left.+\sum_{\chi} \chi \mathcal{R}\left(x^{\prime}, \chi ; x, \chi\right)\right]
\end{aligned}
$$

where $E_{\lambda}$ and $\mu\left(x, x^{\prime}\right)=\mu_{q} \delta\left(x, x^{\prime}\right)$ are Lagrange multipliers introduced to ensure the normalization condition for $\psi_{\lambda ;-}(x ; \chi)$ [see Eq. (5)] and neutron- and proton-number conservation

$$
\sum_{\sigma} \int d \boldsymbol{r} \rho(\boldsymbol{r}, \sigma, q ; \boldsymbol{r}, \sigma, q)=N_{q},
$$

which are introduced through Eq. (28). Applying the variational principle to the functional $F$, we obtain the following set of equations of motion (see Appendix A for details):

$$
\sum_{\chi^{\prime}} \int d x^{\prime} \mathcal{H}\left(x, \chi ; x^{\prime}, \chi^{\prime}\right) \psi_{\lambda ; \eta}\left(x^{\prime} ; \chi^{\prime}\right)=\eta E_{\lambda} \psi_{\lambda ; \eta}(x ; \chi),
$$

where

$$
\mathcal{H}\left(x, \chi ; x^{\prime}, \chi^{\prime}\right)=2 \frac{\delta \mathcal{E}_{\text {ext }}[\mathcal{R}]}{\delta \mathcal{R}\left(x^{\prime}, \chi^{\prime} ; x, \chi\right)}-\chi \delta_{\chi, \chi^{\prime}} \mu\left(x, x^{\prime}\right) .
$$

These equations can also be written in the matrix form

$$
\left(\begin{array}{cc}
h-\mu & \Delta \\
-\Delta^{*} & \mu-h^{*}
\end{array}\right)\left(\begin{array}{c}
\psi_{\lambda ; \eta}^{(+)} \\
\psi_{\lambda ; \eta}^{(-)}
\end{array}\right)=\eta E_{\lambda}\left(\begin{array}{c}
\psi_{\lambda ; \eta}^{(+)} \\
\psi_{\lambda ; \eta}^{(-)}
\end{array}\right),
$$

where $h$ is the single-particle pseudo-Hamiltonian, and $\Delta$ is the pairing field operator,

$$
h\left(x, x^{\prime}\right)=\frac{\delta \mathcal{E}\left[\rho, \tilde{\kappa}, \tilde{\kappa}^{*}\right]}{\delta \rho\left(x^{\prime}, x\right)}, \quad \Delta\left(x, x^{\prime}\right)=-2 \frac{\delta \mathcal{E}\left[\rho, \tilde{\kappa}, \tilde{\kappa}^{*}\right]}{\delta \tilde{\kappa}^{*}\left(x^{\prime}, x\right)},
$$

and $\psi_{\lambda ; \eta}^{( \pm)}=\psi_{\lambda ; \eta}(x ; \pm)$. Obviously, we can consider $E_{\lambda}>0$ [if $E_{\lambda}<0$, the permutation $\psi_{\lambda ;+}(x ; \chi) \leftrightarrow \psi_{\lambda ;-}(x ; \chi)$ is made]. Thus, the Lagrange multipliers $\mu_{q}$ and $E_{\lambda}$ play the role of the chemical potential for nucleons of the type $q$ and the role of the absolute value of the quasiparticle energy, respectively. From Eq. (34) the sense of the indices $\eta$ and $\chi$, which appear in the functions $\psi_{\lambda ; \eta}(x ; \chi)$ introduced in Sec. II, can be easily understood. The index $\eta$ is the sign of the eigenvalue in Eq. (34), and $\chi$ denotes the upper and lower components of the eigenfunctions. In most cases, one of these components is small, and it completely vanishes for the blocked states in the $\mathrm{CB}$ representation (9) of the functions $\psi_{\lambda ; \eta}(x ; \chi)$. However, in general, the functions of the $\mathrm{CB}$ representation, that is, $\breve{\psi}_{\lambda ; \eta}(x ; \chi)$, are not solutions of Eq. (32), i.e., they are not eigenfunctions of the operator $\mathcal{H}$ (see comments in Appendix B).

As can be seen from Eq. (34), the equations of motion of the EDMFT have the same form as those of the HFB theory. However, the EDMFT does not reduce to this theory. First of all, the total energy functional (26) of the EDMFT has a more general form when compared with the HFB energy functional. Actually, only the part $\tilde{\mathcal{E}}$ corresponds to the HFB functional, whereas the term $E_{\mathrm{RC}}$ has, in general, a more complicated and less obvious functional dependence on the DM $\rho$. However, the basic difference between the HFB theory and the EDMFT is determined by the following: The EDMFT is (in principle) an exact theory in the sense that Eqs. (27) are fulfilled. This is ensured by the fact that the functional $E[\rho]$, which enters the term $E_{\mathrm{RC}}$, is defined by Eq. (23) through a set of wave functions $\Psi$ which contains the exact wave function $\Psi_{\mathrm{GS}}$ with a fixed number of particles. In this sense, one can say that the number of particles in the EDMFT is conserved exactly, despite the fact that the auxiliary quantity $\tilde{\kappa}$ (which has the sense of an anomalous DM but does not coincide with $\kappa_{\mathrm{GS}}$ ) takes nonzero values. In this context, the HFB theory can be considered as the phenomenological realization of the EDMFT. The relationship between the EDMFT and HFB approaches is analogous to the relationship between the theory developed in Ref. [9] and the density-dependent Hartree-Fock theory, as discussed in more detail in Ref. [9].

\section{REDUCTION TO THE EXTENDED QUASILOCAL THEORY}

The theory developed in the previous sections is essentially nonlocal as can be seen from Eqs. (34) and (35). The exact solution of these equations is a rather complicated problem. However, one can noticeably simplify the task of solving these equations by reducing the total energy functional $\mathcal{E}\left[\rho, \tilde{\kappa}, \tilde{\kappa}^{*}\right]$ to a quasilocal form following the method described in Ref. [9].

In the particular case of the atomic nucleus, let us introduce a set of local quantities consisting of the local particle $n_{q}$, kinetic-energy $\tau_{q}$, and spin $\boldsymbol{J}_{q}$ densities for neutrons and protons, which are obtained from the nonlocal DM $\rho$ as

$$
n_{q}(\boldsymbol{r})=\sum_{\sigma} \int d x^{\prime} \delta\left(x, x^{\prime}\right) \rho\left(x, x^{\prime}\right)
$$




$$
\begin{aligned}
\tau_{q}(\boldsymbol{r}) & =\sum_{\sigma} \int d x^{\prime} \delta\left(x, x^{\prime}\right)\left(\nabla_{\mathrm{r}} \nabla_{\mathrm{r}^{\prime}}\right) \rho\left(x, x^{\prime}\right), \\
\boldsymbol{J}_{q}(\boldsymbol{r}) & =i \sum_{\sigma} \int d x^{\prime} \delta\left(\boldsymbol{r}-\boldsymbol{r}^{\prime}\right) \delta_{q, q^{\prime}}\left[(\boldsymbol{\sigma})_{\sigma^{\prime}, \sigma} \times \nabla_{\mathrm{r}}\right] \rho\left(x, x^{\prime}\right) .
\end{aligned}
$$

Note that in contrast to the analogous definitions of Ref. [9], the exact DM $\rho$ in this case enters Eqs. (36)-(38) [see remark after Eq. (27)], so the quantities $\tau_{q}$ and $\boldsymbol{J}_{q}$ are the exact (correlated) neutron and proton kinetic-energy and spin densities, respectively. Explicit expressions for these local quantities, which follow from Eqs. (16) and from the representation of the EDM in the form (10), are

$$
\begin{aligned}
n_{q}(\boldsymbol{r})= & \sum_{\sigma} \sum_{\lambda}\left|\psi_{\lambda ;-}(\boldsymbol{r}, \sigma, q ;+)\right|^{2}, \\
\tau_{q}(\boldsymbol{r})= & \sum_{\sigma} \sum_{\lambda}\left|\nabla \psi_{\lambda ;-}(\boldsymbol{r}, \sigma, q ;+)\right|^{2}, \\
\boldsymbol{J}_{q}(\boldsymbol{r})= & i \sum_{\sigma, \sigma^{\prime}} \sum_{\lambda} \psi_{\lambda ;-}^{*}\left(\boldsymbol{r}, \sigma^{\prime}, q ;+\right) \\
& \times\left[(\boldsymbol{\sigma})_{\sigma^{\prime}, \sigma} \times \nabla\right] \psi_{\lambda ;-}(\boldsymbol{r}, \sigma, q ;+) .
\end{aligned}
$$

We can also define the quantities $\varkappa_{q}$, which represent local anomalous densities for each kind of nucleon, as

$$
\begin{aligned}
\varkappa_{q}(\boldsymbol{r}) & =i \sum_{\sigma} \int d x^{\prime} \delta\left(\boldsymbol{r}-\boldsymbol{r}^{\prime}\right) \delta_{q, q^{\prime}}\left(\sigma_{y}\right)_{\sigma^{\prime}, \sigma} \tilde{\kappa}\left(x, x^{\prime}\right) \\
& =i \sum_{\sigma, \sigma^{\prime}} \sum_{\lambda} \psi_{\lambda ;-}^{*}\left(\boldsymbol{r}, \sigma^{\prime}, q ;-\right)\left(\sigma_{y}\right)_{\sigma^{\prime}, \sigma} \psi_{\lambda ;-}(\boldsymbol{r}, \sigma, q ;+) .
\end{aligned}
$$

Let us now introduce three kinds of short notation: $\rho_{\mathrm{QL}}=$ $\left\{n_{n}, n_{p}, \tau_{n}, \tau_{p}, J_{n}, J_{p}\right\}, \varkappa=\left\{\varkappa_{n}, \varkappa_{p}\right\}$, and $\varkappa^{*}=\left\{\varkappa_{n}^{*}, \varkappa_{p}^{*}\right\}$. Using this notation, we define the following quasilocal energy functionals:

$$
\begin{aligned}
\mathcal{E}^{\mathrm{QL} 1}\left[\rho_{\mathrm{QL}}, \tilde{\kappa}, \tilde{\kappa}^{*}\right] & =\inf _{\rho \rightarrow \rho_{\mathrm{QL}}} \mathcal{E}\left[\rho, \tilde{\kappa}, \tilde{\kappa}^{*}\right], \\
\mathcal{E}^{\mathrm{QL} 2}\left[\rho_{\mathrm{QL}}, \varkappa, \varkappa^{*}\right] & =\inf _{\rho \rightarrow \rho_{\mathrm{QL}}} \inf _{\tilde{\kappa} \rightarrow \varkappa \tilde{\kappa}^{*} \rightarrow \varkappa^{*}} \mathcal{E}\left[\rho, \tilde{\kappa}, \tilde{\kappa}^{*}\right] .
\end{aligned}
$$

Note that the mappings $\rho \rightarrow \rho_{\mathrm{QL}}, \tilde{\kappa} \rightarrow \varkappa$, and $\tilde{\kappa}^{*} \rightarrow \varkappa^{*}$ are established according to Eqs. (36)-(38) and (42). From the definitions (44)-(45), it immediately follows that the property (27) is also true for both energy functionals $\mathcal{E}^{\mathrm{QL} 1}$ and $\mathcal{E}^{\mathrm{QL} 2}$. Namely, we have

$$
\inf _{\rho_{\mathrm{QL}}, \tilde{\kappa}, \tilde{\kappa}^{*}} \mathcal{E}^{\mathrm{QL} 1}\left[\rho_{\mathrm{QL}}, \tilde{\kappa}, \tilde{\kappa}^{*}\right]=\inf _{\rho_{\mathrm{QL}}, \varkappa, \varkappa^{*}} \mathcal{E}^{\mathrm{QL} 2}\left[\rho_{\mathrm{QL}}, \varkappa, \varkappa^{*}\right]=E_{\mathrm{GS}} .
$$

In the following, we shall refer to the theories based on the functionals (44) and (45) as the extended quasilocal density functional theories EQLT1 and EQLT2.

The equations of motion for these quasilocal theories have the same matrix form (34) as for the nonlocal theory, but with different definitions of the operators $h$ and $\Delta$. Making use of
Eqs. (35) and (36)-(38), we obtain

$$
\begin{aligned}
h\left(x, x^{\prime}\right)= & \delta_{q, q^{\prime}}\left\{\left[-\nabla_{\mathrm{r}} \frac{\hbar^{2}}{2 m_{q}^{*}(\boldsymbol{r})} \nabla_{\mathrm{r}}+U_{q}(\boldsymbol{r})\right] \delta_{\sigma, \sigma^{\prime}}\right. \\
& \left.-i \mathbf{W}_{q}(\boldsymbol{r}) \cdot\left[\nabla_{\mathrm{r}} \times \boldsymbol{\sigma}\right]_{\sigma, \sigma^{\prime}}\right\} \delta\left(\boldsymbol{r}-\boldsymbol{r}^{\prime}\right),
\end{aligned}
$$

where, for the quasilocal theory EQLT1,

$$
\begin{aligned}
\frac{\hbar^{2}}{2 m_{q}^{*}(\boldsymbol{r})} & =\frac{\delta \mathcal{E}^{\mathrm{QL} 1}}{\delta \tau_{q}(\boldsymbol{r})}, \quad U_{q}(\boldsymbol{r})=\frac{\delta \mathcal{E}^{\mathrm{QL} 1}}{\delta n_{q}(\boldsymbol{r})}, \\
\boldsymbol{W}_{q}(\boldsymbol{r}) & =\frac{\delta \mathcal{E}^{\mathrm{QL} 1}}{\delta \boldsymbol{J}_{q}(\boldsymbol{r})},
\end{aligned}
$$

and analogous expressions with the replacement of $\mathcal{E}^{\mathrm{QL} 1}$ by $\mathcal{E}^{\mathrm{QL} 2}$ in the case of the quasilocal theory EQLT2. The difference between these two versions of the quasilocal approach consists of the definition of the operator $\Delta$. Within the EQLT1, we have

$$
\Delta\left(x, x^{\prime}\right)=-2 \frac{\delta \mathcal{E}^{\mathrm{QL} 1}}{\delta \tilde{\kappa}^{*}\left(x^{\prime}, x\right)},
$$

as in the nonlocal theory; whereas in the case of the EQLT2, the operator $\Delta$ is purely local and is obtained using Eq. (42)

$$
\Delta\left(x, x^{\prime}\right)=-2 i \delta\left(\boldsymbol{r}-\boldsymbol{r}^{\prime}\right) \delta_{q, q^{\prime}}\left(\sigma_{y}\right)_{\sigma, \sigma^{\prime}} \frac{\delta \mathcal{E}^{\mathrm{QL} 2}}{\delta \varkappa_{q}^{*}(\boldsymbol{r})} .
$$

Let us stress that the solution of the equations of motion associated with both EQLT1 and EQLT2 enables us (at least in principle) to calculate the exact values of all the local densities entering the set $\rho_{\mathrm{QL}}$. The theory of Ref. [9] only allows the exact values of the local particle densities $n_{q}(\boldsymbol{r})$ to be found. This difference comes from the different character of the many-fermion wave functions used for building up the energy functional: a Slater-determinant wave function in [9] for which only the Lieb theorem was proved, and a quasiparticle-vacuum wave function in the present paper which enables one, as has been shown, to reproduce an arbitrary nonlocal DM $\rho$. More involved energy functionals including other additional local densities have been proposed recently in Ref. [14]. However, our theory remains valid, and only the local densities related with the normal DM take the exact values, whereas the ones related with the anomalous part of the EDM $\mathcal{R}$ become auxiliary quantities which do not correspond to their exact values.

In comparing our approach with the theory developed in Ref. [3], it should be noted that we do not introduce any external anomalous pair potential as was done in [3]. In our method, the pairing only emerges as a consequence of the interaction between the fermions. In the absence of the interaction, the pairing field $\Delta$ vanishes, which is in agreement with the particle-number conservation condition. The same difference exists between the equations of motion in the EQLT2 case and the Bogoliubov-de Gennes equations [15], in which the pair potential enters, in fact, as an external field (though created initially by the interaction).

It should also be pointed out that the EDMFT in its nonlocal and quasilocal versions can be reduced to the simpler DFT 
plus BCS description of the pairing correlations if the operator $\mathcal{H}$ in Eq. (32) is (or is assumed to be) diagonal in the $\mathrm{CB}$ representation (9) (see the next section). However, in general, this description is not equivalent to that obtained by exactly solving the EDMFT equations of motion (see comments in Appendix B). This reduction of the EDMFT is analogous to the replacement of the HFB equations by coupled Hartree-Fock plus BCS equations, as discussed in Refs. [10,11].

\section{EXTENDED QUASILOCAL THEORY WITHIN BCS APPROXIMATION}

In this section, we consider approximate versions of our EQLT1 and EQLT2 theories which are very easy for numerical applications. First, we extract from the energy functionals $\mathcal{E}^{\mathrm{QL} 1}$ and $\mathcal{E}^{\mathrm{QL} 2}$ a part $\mathcal{E}_{\text {norm }}^{\mathrm{QL}}\left[\rho_{\mathrm{QL}}\right]$ depending only on the normal densities. It is supposed that $\mathcal{E}_{\text {norm }}^{\mathrm{QL}}$ is a fairly good approximation of the functionals $\mathcal{E}^{\mathrm{QL} 1}$ and $\mathcal{E}^{\mathrm{QL} 2}$ in the case when pairing correlations can be neglected. To construct this part of the functionals, we will use the method developed in Ref. [9]. The remaining parts of $\mathcal{E}^{\mathrm{QL} 1}$ and $\mathcal{E}^{\mathrm{QL} 2}$ contain contributions arising from the pairing correlations and will be denoted as $\mathcal{E}_{\text {pair }}^{\mathrm{QL1}}$ and $\mathcal{E}_{\text {pair }}^{\mathrm{QL2}}$. For these parts, we will use the results obtained in density-dependent HFB theory, that is,

$$
\begin{aligned}
\mathcal{E}_{\text {pair }}^{\mathrm{QL} 1}\left[\rho_{\mathrm{QL}}, \tilde{\kappa}, \tilde{\kappa}^{*}\right]= & \frac{1}{4} \int d x_{1} d x_{2} d x_{3} d x_{4} \tilde{\kappa}^{*}\left(x_{1}, x_{2}\right) \\
& \times V^{\mathrm{pp}}\left(x_{1}, x_{2} ; x_{3}, x_{4}\right) \tilde{\kappa}\left(x_{3}, x_{4}\right), \\
\mathcal{E}_{\text {pair }}^{\mathrm{QL} 2}\left[\rho_{\mathrm{QL}}, \varkappa, \varkappa^{*}\right]= & \frac{1}{2} \int d \boldsymbol{r} V^{\mathrm{pp}}(\boldsymbol{r}) \sum_{q} \varkappa_{q}^{*}(\boldsymbol{r}) \varkappa_{q}(\boldsymbol{r}) .
\end{aligned}
$$

Here $V^{\mathrm{pp}}\left(x_{1}, x_{2} ; x_{3}, x_{4}\right)$ and $V^{\mathrm{pp}}(\boldsymbol{r})$ are finite-range and zero-range effective interactions, respectively, in the particleparticle (p-p) channel. These interactions can also depend on the local density $n(\boldsymbol{r})=n_{n}(\boldsymbol{r})+n_{p}(\boldsymbol{r})$ [see Eq.(69) below]. Also notice that in general the interactions $V^{\mathrm{pp}}\left(x_{1}, x_{2} ; x_{3}, x_{4}\right)$ and $V^{\mathrm{pp}}(\boldsymbol{r})$ can be quite different. So, we have

$$
\begin{aligned}
\mathcal{E}^{\mathrm{QL} 1}\left[\rho_{\mathrm{QL}}, \tilde{\kappa}, \tilde{\kappa}^{*}\right] & =\mathcal{E}_{\text {norm }}^{\mathrm{QL}}\left[\rho_{\mathrm{QL}}\right]+\mathcal{E}_{\text {pair }}^{\mathrm{QL} 1}\left[\rho_{\mathrm{QL}}, \tilde{\kappa}, \tilde{\kappa}^{*}\right], \\
\mathcal{E}^{\mathrm{QL} 2}\left[\rho_{\mathrm{QL}}, \varkappa, \varkappa^{*}\right] & =\mathcal{E}_{\text {norm }}^{\mathrm{QL}}\left[\rho_{\mathrm{QL}}\right]+\mathcal{E}_{\text {pair }}^{\mathrm{QL} 2}\left[\rho_{\mathrm{QL}}, \varkappa, \varkappa^{*}\right] .
\end{aligned}
$$

In the application of our approach, we calculate the pairing parts of the energy functionals, i.e., $\mathcal{E}_{\text {pair }}^{\mathrm{QL1}}$ and $\mathcal{E}_{\text {pair }}^{\mathrm{QL} 2}$, within the framework of the state-dependent version of the BCS approximation (see, e.g., Ref. [10]). This approximation implies that the antisymmetric operator of the pairing field $\Delta$ [gap, see Eqs. (49) and (50)] is supposed to have the canonical form in the representation of the eigenfunctions of singleparticle pseudo-Hamiltonian $h$ defined by Eqs. (47) and (48). In this case, the solutions $\psi_{\lambda ; \eta}^{( \pm)}=\psi_{\lambda ; \eta}(x ; \pm)$ of the equation of motion (34) are the functions of the CB representation (9) in which the functions $\tilde{\phi}_{\lambda}(x)$ are eigenfunctions of the operator $h$. In particular, for the spherically symmetric system, we have

$\psi_{\lambda ;+}(x ;+)=u_{\lambda} \tilde{\phi}_{\lambda}(x), \psi_{\lambda ;+}(x ;-)=(-1)^{l+j+m} v_{\lambda} \tilde{\phi}_{\lambda}^{*}(x)$, $\left.\psi_{\lambda ;-}(x ;-)=u_{\lambda} \tilde{\phi}_{\lambda}^{*}(x), \psi_{\lambda ;-}(x ;+)=(-1)^{l+j+m} v_{\lambda} \widetilde{\phi}_{\bar{\lambda}}(x),\right\}$ where $\lambda=\{(\lambda), m\}, \bar{\lambda}=\{(\lambda),-m\},(\lambda)=\left\{q_{\lambda}, n, l, j\right\}$, and $m$ is the projection of the total angular momentum $j$. The choice of the phase factors is determined by the formulas

$$
\begin{aligned}
& \tilde{\phi}_{\lambda}(x)=\delta_{q_{\lambda}, q} R_{(\lambda)}(\boldsymbol{r}) \sum_{\mu}\left(l \mu \frac{1}{2} \sigma \mid j m\right) Y_{l \mu}(\boldsymbol{n}), \\
& \tilde{\phi}_{\lambda}(x)=(-1)^{l+j+m+\frac{1}{2}+\sigma} \tilde{\phi}_{\bar{\lambda}}^{*}(\bar{x}),
\end{aligned}
$$

where $\boldsymbol{n}=r / r, \bar{x}=\{r,-\sigma, q\}$. Thus, Eq. (34) is reduced to the equation

$$
h \tilde{\phi}_{\lambda}=\varepsilon_{\lambda} \tilde{\phi}_{\lambda}
$$

determining single-particle energies $\varepsilon_{\lambda}$, and to the gap equation which for spherically symmetric systems has the form

$$
\Delta_{\lambda}=-\sum_{\left(\lambda^{\prime}\right)} \frac{2 j_{\lambda^{\prime}}+1}{4 \pi} V_{\left(\lambda \lambda^{\prime}\right)}^{\mathrm{pp}} \frac{\Delta_{\lambda^{\prime}}}{2 E_{\lambda^{\prime}}},
$$

where $V_{\left(\lambda \lambda^{\prime}\right)}^{\mathrm{pp}}$ is the reduced matrix element of the effective interaction in the p-p channel. The quasiparticle energy $E_{\lambda}$ and the $u_{\lambda}$ and $v_{\lambda}$ amplitudes entering the above equations are determined by the usual formulas of BCS theory:

$$
\begin{aligned}
E_{\lambda} & =\sqrt{\left(\varepsilon_{\lambda}-\mu_{q_{\lambda}}\right)^{2}+\Delta_{\lambda}^{2}}, \\
u_{\lambda} & =\sqrt{\frac{1}{2}\left(1+\frac{\varepsilon_{\lambda}-\mu_{q_{\lambda}}}{E_{\lambda}}\right)}, \\
v_{\lambda} & =\operatorname{sgn}\left(\Delta_{\lambda}\right) \sqrt{\frac{1}{2}\left(1-\frac{\varepsilon_{\lambda}-\mu_{q_{\lambda}}}{E_{\lambda}}\right)} .
\end{aligned}
$$

In terms of the above-defined quantities, the values of the functionals $\mathcal{E}_{\text {pair }}^{\mathrm{QL} 1}$ and $\mathcal{E}_{\text {pair }}^{\mathrm{QL2}}$ in the equilibrium point (pairing energies) are determined by the ansatz

$$
E_{\text {pair }}^{\mathrm{BCS}}=-\sum_{(\lambda)}\left(2 j_{\lambda}+1\right) \frac{\Delta_{\lambda}^{2}}{4 E_{\lambda}},
$$

which is the same for both functionals.

Notice that in principle the equation of motion (34) should be solved exactly, without further approximations. However, for pairing calculations of a wide range of nuclei not too far from the $\beta$-stability line, the simpler BCS approach can be sufficient in order to describe their ground-state energies. For these nuclei, the Fermi level lies appreciably below zero; consequently, the levels around it, which mainly contribute to the pairing correlations, are also well bound, which allows one to avoid the problems related with the continuum which have been described in detail in Ref. [11].

Equations (58) and (59) with the condition (31) are solved self-consistently. The normal densities entering these equations through Eqs. (47) and (48) and through the density dependence of the effective interaction in the $p-p$ channel [see, for instance, Eq. (69) below in the case of a contact density-dependent force] are calculated according to Eqs. (36) $-(38)$ and (15) (where $\tilde{\rho}=\rho, \tilde{v}_{\lambda}=v_{\lambda}$ ). The 
anomalous densities have the form

$$
\begin{gathered}
\tilde{\kappa}\left(x, x^{\prime}\right)=\sum_{\lambda}(-1)^{l+j+m} u_{\lambda} v_{\lambda} \tilde{\phi}_{\bar{\lambda}}(x) \tilde{\phi}_{\lambda}\left(x^{\prime}\right), \\
\varkappa_{q}(r)=\sum_{(\lambda)} \delta_{q_{\lambda}, q} \frac{2 j_{\lambda}+1}{4 \pi} u_{\lambda} v_{\lambda} R_{(\lambda)}^{2}(r) .
\end{gathered}
$$

To illustrate the approach presented in this paper, we consider the energy functional based on the effective Gogny forces [16] whose parameters are adjusted to describe the the ground-state properties of some spherical nuclei. The method of construction of the functional $\mathcal{E}_{\text {norm }}^{\mathrm{QL}}$ in this case is described in detail in Ref. [9]. For the pairing parts of the energy functionals, we use Eqs. (51) and (52) for the EQLT1 and EQLT2 theories, correspondingly. The reduced matrix elements of the effective interaction in the p-p channel also have different forms within EQLT1 and EQLT2. For EQLT1 in the case of a standard Gogny force, it can be written as

$$
\begin{aligned}
V_{\left(\lambda \lambda^{\prime}\right)}^{\mathrm{pp}}= & \delta_{q_{\lambda}, q_{\lambda^{\prime}}} 2 \pi \sum_{i=1}^{2} \sum_{L} V_{i, L\left(\lambda \lambda^{\prime}\right)}\left(l 0 l^{\prime} 0 \mid L 0\right)^{2} \\
& \times\left\{\left(W_{i}-B_{i}-H_{i}+M_{i}\right)+\left(W_{i}+B_{i}-H_{i}-M_{i}\right)\right. \\
& \times \frac{8(j-l)\left(j^{\prime}-l^{\prime}\right)}{(2 j+1)\left(2 j^{\prime}+1\right)}[l(l+1) \\
& \left.\left.+l^{\prime}\left(l^{\prime}+1\right)-L(L+1)\right]\right\}
\end{aligned}
$$

where

$$
\begin{aligned}
V_{i, L\left(\lambda \lambda^{\prime}\right)}= & \int_{0}^{\infty} d r_{1} r_{1}^{2} \int_{0}^{\infty} d r_{2} r_{2}^{2} v_{i, L}\left(r_{1}, r_{2}\right) \\
& \times R_{(\lambda)}\left(r_{1}\right) R_{\left(\lambda^{\prime}\right)}\left(r_{1}\right) R_{(\lambda)}\left(r_{2}\right) R_{\left(\lambda^{\prime}\right)}\left(r_{2}\right), \\
v_{i, L}\left(r_{1}, r_{2}\right)= & i_{L}\left(2 r_{1} r_{2} / \mu_{i}^{2}\right) e^{-\left(r_{1}^{2}+r_{2}^{2}\right) / \mu_{i}^{2}},
\end{aligned}
$$

$W_{i}, B_{i}, H_{i}, M_{i}$, and $\mu_{i}$ are the parameters of the finite-range density-independent part of the Gogny force (notice that the zero-range part of the Gogny force does not contribute to the pairing channel because of the particular value of the $x_{0}$ parameter, which is equal to unity in the standard parametrizations of this force, see Ref. [16]), and $i_{L}(z)$ are the modified spherical Bessel functions.

For the EQLT2, we have

$$
V_{\left(\lambda \lambda^{\prime}\right)}^{\mathrm{pp}}=\delta_{q_{\lambda}, q_{\lambda^{\prime}}} \int_{0}^{\infty} d r r^{2} R_{(\lambda)}^{2}(r) R_{\left(\lambda^{\prime}\right)}^{2}(r) V^{\mathrm{pp}}(n(r)) .
$$

For the effective interaction in Eqs. (52) and (68), we use the density-dependent form proposed by Bertsch and Esbensen [17]:

$$
V^{\mathrm{pp}}(n(\boldsymbol{r}))=\frac{v_{0}}{2}\left[1-\eta\left(\frac{n(\boldsymbol{r})}{n_{0}}\right)^{\alpha}\right], \quad n_{0}=\frac{2}{3 \pi^{2}} k_{F}^{3} .
$$

The numerical values of the parameters $v_{0}=-481 \mathrm{MeV}$, $\eta=0.45, \alpha=0.47$, and $k_{F}=1.35 \mathrm{fm}^{-1}$ in Eq. (69) are taken from Ref. [18]. Some of these parameters, namely, $\eta$ and $\alpha$ which fix the density dependence of the pairing interaction, were fitted to reproduce the density dependence of the pairing gap in nuclear matter provided by the Gogny D1 force [18]. Thus, from this perspective, the finite-range density-independent Gogny interaction in the p-p channel is equivalent to the zero-range density-dependent force (69) [19]. Consequently, the calculation of some quantities such as ground-state (binding) energies or average gaps obtained using our approach with the pairing correlations included at the BCS level and the Gogny interaction allows us to compare explicitly the EQLT1 (nonlocal pairing field) and EQLT2 (local pairing field) approximations between themselves as well as to compare them with the full HFB theory.

The zero-range pairing force in Eq. (69) must be supplemented by a cutoff energy $\varepsilon_{C}$ in order to avoid the divergence of the gap equation. This cutoff energy is, actually, a fourth parameter of the force. In our calculation of tin isotopes, the pairing window includes all the neutron single-particle states from the bottom of the single-particle potential well up to $10 \mathrm{MeV}$ above zero. In the case of the considered tin isotopes this leads to a cutoff energy $\varepsilon_{C}$ of about $65 \mathrm{MeV}$ (measured from the bottom of the single-particle potential), which is roughly similar to the $\varepsilon_{C}=60 \mathrm{MeV}$ used in Ref. [18] in the pairing calculation in nuclear matter. To account for the positive energy single-particle levels needed in the calculation and to avoid the problems encountered in the treatment of the continuum [11], we follow the strategy described in Ref. [20] where the resonant levels [11], which discretize the continuum, are very well simulated by the so-called quasibound energy levels, i.e., levels which are bound because of their centrifugal barrier and whose wave functions are well localized in spite of their positive energy. Furthermore, to solve the gap equation using the finite-range Gogny force pairing matrix elements (65) (i.e., within the EQLT1 theory), although this equation does not diverge, it is necessary to take into account single-particle levels which lie in the continuum even for nuclei for which the Fermi level is negative and far from zero, as in the case of nuclei considered in Table I. We have found that by taking all the possible

TABLE I. Ground-state energies (in $\mathrm{MeV}$ ) of some even Sn isotopes calculated using the exact HF method (without pairing correlations, see Ref. [16]) and the quasilocal density functional theory (QLDFT) developed in [9], and those calculated within the exact HFB theory [16] and within the density functional theories EQLT1 and EQLT2 plus BCS (see text). All results were obtained using the Gogny D1 force; and, as in [16], the two-body part of the center-of-mass correction was omitted.

\begin{tabular}{lrrrrrr}
\hline \hline & HF [16] & $\begin{array}{c}\text { QLDFT } \\
{[9]}\end{array}$ & $\begin{array}{l}\text { HFB } \\
{[16]}\end{array}$ & EQLT1 & EQLT2 \\
\hline${ }^{112} \mathrm{Sn}$ & -948.301 & -949.504 & -953.065 & -953.064 & -954.559 \\
${ }^{114} \mathrm{Sn}$ & -968.403 & -969.709 & -971.434 & -971.538 & -972.972 \\
${ }^{116} \mathrm{Sn}$ & -985.701 & -987.205 & -988.939 & -989.184 & -990.592 \\
${ }^{118} \mathrm{Sn}$ & -1002.164 & -1003.611 & -1005.553 & -1005.970 & -1007.417 \\
${ }^{120} \mathrm{Sn}$ & -1018.898 & -1020.229 & -1021.310 & -1021.967 & -1023.481 \\
${ }^{122} \mathrm{Sn}$ & -1032.187 & -1033.856 & -1036.295 & -1037.288 & -1038.837 \\
${ }^{124} \mathrm{Sn}$ & -1045.901 & -1047.783 & -1050.605 & -1052.045 & -1053.533 \\
\hline \hline
\end{tabular}


quasibound levels up to $60 \mathrm{MeV}$ above the Fermi level, the BCS gap equation becomes stable in the EQLT1 calculations performed with the D1 and D1S forces. The energies of the ${ }^{112} \mathrm{Sn}-{ }^{124} \mathrm{Sn}$ chain of isotopes obtained within our density functional theories EQLT1 and EQLT2 plus BCS are presented in Table I. These results are compared with the exact HFB energies reported in Ref. [16]. This table also displays the energies obtained from our quasilocal DFT without pairing correlations [9] compared with the exact HF results from Ref. [16]. All these HF, HFB, and DFT calculations were performed using the Gogny D1 force, and the two-body part of the center-of-mass correction has been omitted (see Table V of Ref. [16] for details). From this comparison, we see that at the pure mean-field level, the energies computed with our quasilocal energy density functional reproduce the exact HF results with relative differences less than $0.2 \%$, similar to the differences found in the DFT calculations in magic nuclei reported in Ref. [9]. Thus, our local mean field simulates very well the nonlocal mean field used in the full HF calculations. Furthermore, we can also see that the exact HFB values are reproduced very precisely by our quasilocal EQLT1+BCS approach, even slightly better than at the pure mean-field (HF) level. When the zero-range density-dependent force (69) is used in the p-p channel, the EQLT2+BCS results agree nicely with the HFB results, in this case the differences being smaller than $0.3 \%$. From these results, we see that EQLT1 and EQLT2 theories are well suited for dealing with pairing correlations at least concerning ground-state energies of open shell nuclei not very far from the $\beta$-stability valley.

The ground-state energies of the even $\mathrm{Sn}$ isotopes with neutron numbers between $N=50$ and $N=82$ calculated with the EQLT2 functional [i.e., by using the zero-range density-dependent force (69)] are shown in Table II together with the exact HFB results [21] and the experimental values [22]. In these calculations, the Gogny D1S force [23] was used. From these results, we see that the EQLT2+BCS calculations using the local density-dependent version of the Gogny pairing force (69) nicely reproduce again the HFB results with an accuracy better than $0.4 \%$. In Table II, we also show the BCS pairing energies for these nuclei which are defined by Eq. (62). In the same table, we also display the spectral gaps [24] calculated within the EQLT2+BCS approach. The latter quantity is defined, in agreement with Ref. [11], by averaging the state-dependent gaps which are the solution of the set of Eqs. (59), as follows:

$$
\bar{\Delta}_{q}=\frac{\sum_{(\lambda)} \delta_{q_{\lambda}, q}\left(2 j_{\lambda}+1\right) v_{\lambda}^{2} \Delta_{\lambda}}{\sum_{(\lambda)} \delta_{q_{\lambda}, q}\left(2 j_{\lambda}+1\right) v_{\lambda}^{2}},
$$

where the sums in Eq. (70) run over all the states $(\lambda)$ (see before) contained in the pairing window. This average gap (70) can be considered as a possible estimate of the strength of the pairing correlations [24].

To perform a clear comparison between EQLT1 and EQLT2 theories including pairing within the BCS approximation, we display in Fig. 1 the two-neutron separation energies of isotopes in the ${ }^{100} \mathrm{Sn}-{ }^{132} \mathrm{Sn}$ range calculated within these approaches. The corresponding HFB results from Ref. [21] as
TABLE II. Ground-state energies (in $\mathrm{MeV}$ ) of $\mathrm{Sn}$ isotopes calculated within EQLT2+BCS approximation compared with experimental data from Ref. [22] and with the exact HFB results. Pairing energies within BCS approximation and the average pairing gaps for neutrons are listed in the last two columns. Results were obtained using Gogny D1S force [23].

\begin{tabular}{lcccrc}
\hline \hline & Experiment & HFB & EQLT2 & $E_{\text {pair }}^{\text {BCS }}$ & $\bar{\Delta}_{n}$ \\
\hline${ }^{100} \mathrm{Sn}$ & -824.800 & -831.276 & -827.125 & 0.000 & 0.00 \\
${ }^{102} \mathrm{Sn}$ & -849.090 & -853.512 & -849.971 & -5.958 & 1.31 \\
${ }^{104} \mathrm{Sn}$ & -871.890 & -874.888 & -871.850 & -9.989 & 1.68 \\
${ }^{106} \mathrm{Sn}$ & -893.870 & -895.463 & -892.794 & -13.153 & 1.91 \\
${ }^{108} \mathrm{Sn}$ & -914.626 & -915.297 & -912.862 & -15.805 & 2.07 \\
${ }^{110} \mathrm{Sn}$ & -934.571 & -934.424 & -932.146 & -17.768 & 2.17 \\
${ }^{112} \mathrm{Sn}$ & -953.531 & -952.857 & -950.712 & -18.945 & 2.22 \\
${ }^{114} \mathrm{Sn}$ & -971.574 & -970.596 & -968.592 & -19.423 & 2.22 \\
${ }^{116} \mathrm{Sn}$ & -988.684 & -987.636 & -985.794 & -19.386 & 2.19 \\
${ }^{118} \mathrm{Sn}$ & -1004.954 & -1004.018 & -1002.325 & -19.013 & 2.15 \\
${ }^{120} \mathrm{Sn}$ & -1020.546 & -1019.778 & -1018.198 & -18.379 & 2.09 \\
${ }^{122} \mathrm{Sn}$ & -1035.529 & -1034.978 & -1033.442 & -17.412 & 2.01 \\
${ }^{124} \mathrm{Sn}$ & -1049.963 & -1049.655 & -1048.090 & -15.924 & 1.91 \\
${ }^{126} \mathrm{Sn}$ & -1063.889 & -1063.836 & -1062.173 & -13.691 & 1.75 \\
${ }^{128} \mathrm{Sn}$ & -1077.345 & -1077.538 & -1075.715 & -10.491 & 1.52 \\
${ }^{130} \mathrm{Sn}$ & -1090.293 & -1090.763 & -1088.784 & -6.416 & 1.18 \\
${ }^{132} \mathrm{Sn}$ & -1102.851 & -1103.496 & -1101.182 & 0.000 & 0.00 \\
\hline \hline
\end{tabular}

well as the experimental values are also displayed. From this figure, we can see the excellent agreement between EQLT1 and EQLT2 theories. As discussed above, the quasilocal density functional theory, together with the pairing correlations at BCS level, reproduces very accurately the HFB results independently of the approach (EQLT1 or EQLT2) used for the pairing field. From Tables I and II, we can see that the total energies of the analyzed $\mathrm{Sn}$ isotopes provided by both the EQLT1 and EQLT2 theories including pairing at BCS level are shifted by an almost constant value of $2-3 \mathrm{MeV}$ with respect

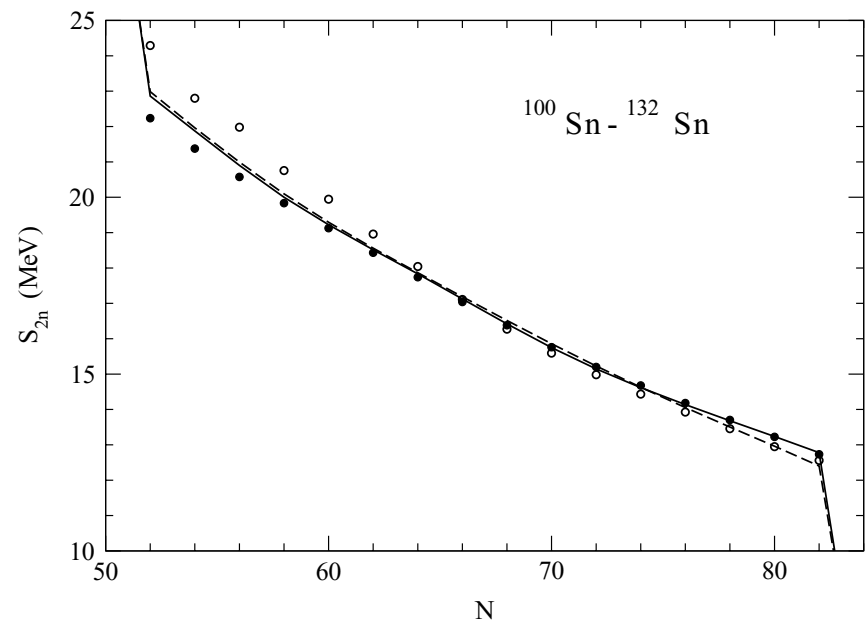

FIG. 1. Two-neutron separation energies of isotopes in the ${ }^{100} \mathrm{Sn}-$ ${ }^{132} \mathrm{Sn}$ range. EQLT1 and EQLT2 plus BCS results are displayed by the solid and broken curves, correspondingly. HFB results from Ref. [21] (filled circles) and experimental values (open circles) are also shown. 


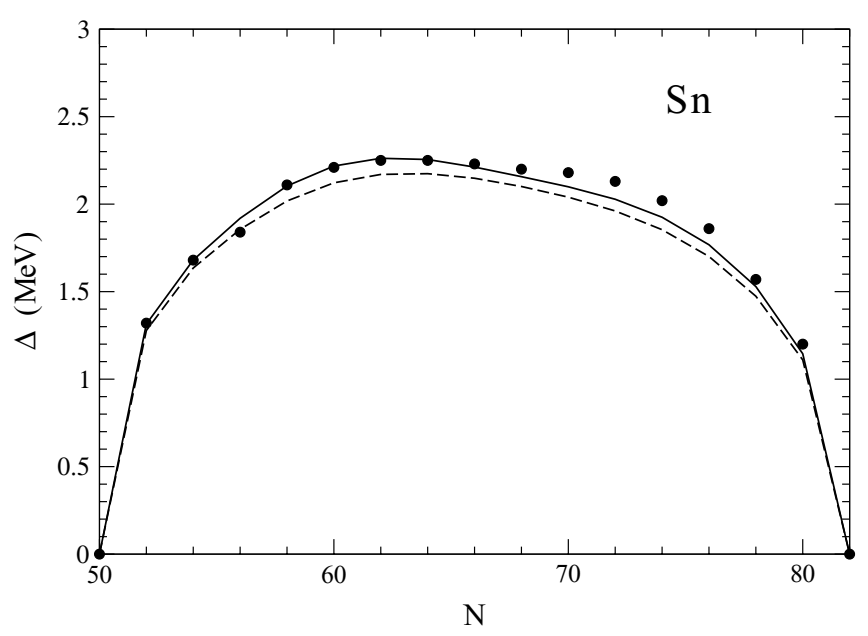

FIG. 2. Average pairing gaps obtained from the EQLT2+BCS (solid line) and EQLT1+BCS calculations (broken line) compared to the HFB results from Ref. [11].

to the HFB values. This difference is similar to the one found at the pure HF level (see Table I). Thus this shift in the total binding energies is due mainly to the differences between the quasilocal and the nonlocal mean fields (see Ref. [9] for details) and is strongly suppressed in the calculation of the two-neutron separation energies, finding almost a perfect agreement between the corresponding quasilocal plus BCS and full HFB results. In adddition, we can also see that the Gogny D1S force taking into account pairing correlations nicely reproduces the experimental two-neutron separation energies of $\mathrm{Sn}$ isotopes with the number of neutrons $N$ between 50 and 82.

In Fig. 2, the values of the average pairing gaps (70) of the ${ }^{100} \mathrm{Sn}-{ }^{132} \mathrm{Sn}$ even isotopes obtained from BCS calculations using EQLT1 and EQLT2 are displayed together with the HFB gaps extracted from Fig. 22 of Ref. [11]. From the Fig. 2, we can see that the average BCS pairing gaps predicted by EQLT1 and EQLT2 theories almost coincide, and they reproduce very well the corresponding HFB values. This fact points out again that for these $\mathrm{Sn}$ isotopes, which lie close to the $\beta$-stability line, the BCS treatment of pairing correlations can simulate, almost perfectly, the more fundamental, but more cumbersome, full HFB calculations.

In Fig. 3, we compare the binding energies per particle, $B / A$, obtained from the results presented in Table II for the HFB and EQLT2 approaches. Both methods obtain the maximum binding energy per particle for the isotope ${ }^{116} \mathrm{Sn}$, in agreement with experiment. The EQLT2 underpredicts $B / A$ for ${ }^{116} \mathrm{Sn}$ by $0.016 \mathrm{MeV}$. We shift the results of the density matrix functional approach by that amount. One finds that slope of the $B / A$ curve representing the HFB results is reproduced better for $\mathrm{Sn}$ isotopes with neutron numbers larger than 116 as compared to those isotopes in the vicinity of ${ }^{100} \mathrm{Sn}$. For ${ }^{100} \mathrm{Sn}$, the deviation between the values for $B / A$ obtained by both methods is less than the discrepancy between the HFB value and experiment. We conclude that the density matrix functional theory is a viable tool for systematic investigations of binding energies of nuclei.

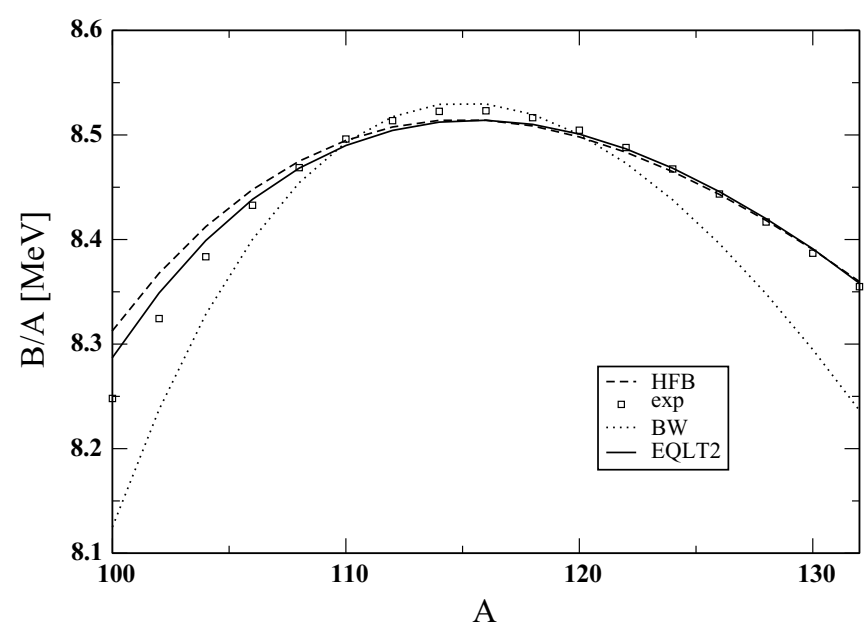

FIG. 3. Binding energies per particle $B / A$ for $\mathrm{Sn}$ isotopes obtained from the results presented in Table II. HFB and EQLT2+BCS results are presented by the broken and solid curves, correspondingly. Experimental data (squares) and results from the Bethe-Weizsäcker formula (dotted curve) are also displayed.

\section{SUMMARY AND CONCLUSIONS}

We have extended the recently developed quasilocal density functional theory [9] to include pairing correlations. This new approach named the extended density matrix functional theory (EDMFT) is based on an extended density matrix (EDM) formalism. The EDM $\mathcal{R}$ contains the normal density matrix $\rho$ as a block and an auxiliary quantity $\tilde{\kappa}$ as another block, which has the sense of an anomalous density matrix but does not coincide with the exact $\kappa$ in general. The matrix $\tilde{\kappa}$ is chosen in such a way that the equality $\mathcal{R}^{2}=\mathcal{R}$ is fulfilled. The EDM which possesses this property can be easily constructed for a given density matrix $\rho$ using the canonical basis in which $\rho$ is diagonal. It has been shown that for an arbitrary density matrix $\rho$, corresponding to some interacting time-reversal invariant fermion system, there is a quasiparticle-vacuum wave function $\tilde{\Psi}$ and an EDM $\mathcal{R}$ such that the following many-to-one mappings take place: $\tilde{\Psi} \rightarrow \mathcal{R} \rightarrow \rho$. This statement can be considered as a generalization of the Lieb theorem. Using the connection between $\mathcal{R}$ and $\rho$, we have defined the total energy functional as an extended functional of $\mathcal{R}$. It has been proved that its minimum value is equal to the exact groundstate energy of the considered system. This extended energy functional is reduced further to a quasilocal form. Thus, in the corresponding final equations of motion, the single-particle pseudo-Hamiltonian $h$ and the pairing field $\Delta$ are both (quasi) local. Although the equations of motion in our theory have the same form as those of the Hartree-Fock-Bogoliubov (HFB) theory, the main difference is that the EDMFT is, in principle, an exact theory in the sense that the true ground-state energy can be reached for the true normal density matrix $\rho$. As an illustration of the practical realizability of our approach, the quasilocal version of the EDMFT has been applied to describe the ground-state energies of the chain of tin isotopes. A very good agreement with the exact HFB results and with experimental data has been obtained. Finally, notice that the 
general formalism developed in this work, which introduces the pairing correlations in the DFT, has been discussed in the particular case of the atomic nucleus, but it can be easily applied to other Fermi systems with a fixed number of particles.

\section{ACKNOWLEDGMENTS}

The authors are indebted to N. Sandulescu for useful discussions and to B. Nerlo-Pomorska for providing us the HFB results computed with the D1S version of the Gogny force. V.I.T. thanks the Institut für Kernphysik at the Forschungszentrum Jülich for hospitality during the completion of this work. S.K. and V.I.T. acknowledge financial support from the Deutsche Forschungsgemeinschaft under Grant No. 436 RUS 113/806/0-1 and from the Russian Foundation for Basic Research under Grant No. 05-02-04005-DFG_a. V.B.S. and V.I.T. acknowledge financial support from the Russian Ministry of Education under Grant No. E02-3.3-463. X.V. acknowledges financial support from DGI and FEDER (Spain) under Grant No. FIS2005-03142 and from DGR (Generalitat de Catalunya) under Grant No. 2005SGR-00343.

\section{APPENDIX A}

In this Appendix, the equations of motion of the EDMFT are derived. Varying the functional $F$ defined by Eq. (30) and taking into account Eq. (10), we obtain

$$
\begin{aligned}
\delta F= & \sum_{\lambda, \chi, \chi^{\prime}} \int d x d x^{\prime}\left\{\frac{\delta \mathcal{E}_{\mathrm{ext}}[\mathcal{R}]}{\delta \mathcal{R}\left(x^{\prime}, \chi^{\prime} ; x, \chi\right)}\right. \\
& \left.-\frac{1}{2} \delta_{\chi, \chi^{\prime}}\left[\chi \mu\left(x, x^{\prime}\right)-\delta\left(x, x^{\prime}\right) E_{\lambda}\right]\right\} \\
& \times\left[\psi_{\lambda ;-}\left(x^{\prime} ; \chi^{\prime}\right) \delta \psi_{\lambda ;-}^{*}(x ; \chi)+\psi_{\lambda ;-}^{*}(x ; \chi)\right. \\
& \left.\times \delta \psi_{\lambda ;-}\left(x^{\prime} ; \chi^{\prime}\right)\right]=0 .
\end{aligned}
$$

This leads to the following equation of motion:

$$
\sum_{\chi^{\prime}} \int d x^{\prime} \mathcal{H}\left(x, \chi ; x^{\prime}, \chi^{\prime}\right) \psi_{\lambda ;-}\left(x^{\prime} ; \chi^{\prime}\right)=-E_{\lambda} \psi_{\lambda ;-}(x ; \chi),
$$

where the operator $\mathcal{H}$ is defined by Eq. (33). Equation (A2) formally defines only half of the complete set of the eigenfunctions of $\mathcal{H}$. In order to define a second half, let us note first that if the following equalities are fulfilled

$$
\begin{aligned}
\mathcal{H}\left(x, \chi ; x^{\prime}, \chi^{\prime}\right) & =-\mathcal{H}\left(x^{\prime},-\chi^{\prime} ; x,-\chi\right) \\
& =\mathcal{H}^{*}\left(x^{\prime}, \chi^{\prime} ; x, \chi\right),
\end{aligned}
$$

then the functions $\psi_{\lambda ;+}(x ; \chi)$, defined through the functions $\psi_{\lambda ;-}(x ; \chi)$ using the condition (7), are also the eigenfunctions of $\mathcal{H}$. In this case, the complete set of eigenfunctions of $\mathcal{H}$ is divided into two equal parts with eigenvalues $+E_{\lambda}$ and $-E_{\lambda}$. On the other hand, if the condition (7) is fulfilled, and if the set of functions $\psi_{\lambda ;-}(x ; \chi)$ is taken as half of the complete set $\left\{\psi_{\lambda ; \pm}(x ; \chi)\right\}$, then Eq. (12) is true; and it follows from Eq. (33) that Eqs. (A3) are also fulfilled. Consequently, properties (7) and (A3) follow from each other, and there exists a solution of the equations of motion which possesses both properties. Thus, setting Eq. (7) to be satisfied, we actually choose a solution which has the symmetry defined by this equation without imposing additional constraints on the variational procedure. In this case, using Eqs. (7) and (A3), we go from (A2) to Eq. (32), which represents a complete set of the equations of motion.

\section{APPENDIX B}

In this Appendix, the relationship between the eigenfunctions of the operators $\mathcal{R}$ and $\mathcal{H}$ is analyzed. First of all, notice that from Eqs. (10) and (5), it follows that

$$
\sum_{\chi^{\prime}} \int d x^{\prime} \mathcal{R}\left(x, \chi ; x^{\prime}, \chi^{\prime}\right) \psi_{\lambda ; \eta}\left(x^{\prime} ; \chi^{\prime}\right)=\delta_{\eta,-} \psi_{\lambda ; \eta}(x ; \chi) \text {. }
$$

Thus, the functions $\psi_{\lambda ; \eta}(x ; \chi)$ are eigenfunctions of the operator $\mathcal{R}$. Obviously, the set of eigenfunctions of $\mathcal{R}$ is determined by a given $\mathcal{R}$ up to an arbitrary unitary transformation of the type Eq. (8). Consequently, the functions $\breve{\psi}_{\lambda ; \eta}(x ; \chi)$ defined by Eqs. (9) are also eigenfunctions of the operator $\mathcal{R}$. On the other hand, from Eqs. (B1) and (32) it follows that there is at least one set of eigenfunctions [namely, the set $\left\{\psi_{\lambda ; \eta}(x ; \chi)\right\}$ ] which is common for both operators $\mathcal{R}$ and $\mathcal{H}$. However, from these equations it does not follow that any eigenfunction of $\mathcal{R}$, and, in particular, $\breve{\psi}_{\lambda ; \eta}(x ; \chi)$, will also be an eigenfunction of $\mathcal{H}$. Indeed, while a set of eigenfunctions of $\mathcal{R}$ is determined up to an arbitrary unitary transformation (8), this is not true for the eigenfunctions of $\mathcal{H}$, since Eqs. (32) and (34) are not covariant under this transformation. Consequently, if we use an arbitrarily given representation of the eigenfunctions of the operator $\mathcal{R}$, in particular the $\mathrm{CB}$ representation (9), an additional nontrivial $C$ transformation, which does not change $\mathcal{R}$, is generally required to diagonalize $\mathcal{H}$ (see Refs. [10,11] for details).
[1] P. Hohenberg and W. Kohn, Phys. Rev. 136, B864 (1964).

[2] W. Kohn and L. J. Sham, Phys. Rev. 140, A1133 (1965).

[3] L. N. Oliveira, E. K. U. Gross, and W. Kohn, Phys. Rev. Lett. 60, 2430 (1988).

[4] N. N. Lathiotakis, M. A. L. Marques, M. Lüders, L. Fast, and E. K. U. Gross, Int. J. Quantum Chem. 99, 790 (2004).

[5] M. Lüders, M. A. L. Marques, N. N. Lathiotakis, A. Floris,
G. Profeta, L. Fast, A. Continenza, S. Massidda, and E. K. U. Gross, Phys. Rev. B 72, 024545 (2005).

[6] S. A. Fayans, S. V. Tolokonnikov, E. L. Trykov, and D. Zawischa, Nucl. Phys. A676, 49 (2000).

[7] A. Bulgac, Phys. Rev. C 65, 051305(R) (2002).

[8] T. L. Gilbert, Phys. Rev. B 12, 2111 (1975).

[9] V. B. Soubbotin, V. I. Tselyaev, and X. Viñas, Phys. Rev. C 67, 014324 (2003). 
[10] P. Ring and P. Schuck, The Nuclear Many-Body Problem (Springer-Verlag, New York, 1980).

[11] J. Dobaczewski, W. Nazarewicz, T. R. Werner, J. F. Berger, C. R. Chinn, and J. Dechargé, Phys. Rev. C 53, 2809 (1996).

[12] C. Bloch and A. Messiah, Nucl. Phys. 39, 95 (1962).

[13] E. H. Lieb, Int. J. Quantum Chem. 24, 243 (1983).

[14] E. Perlińska, S. G. Rohoziński, J. Dobaczewski, and W. Nazarewicz, Phys. Rev. C 69, 014316 (2004).

[15] P. G. de Gennes, Superconductivity of Metals and Alloys (Benjamin, New York, 1966).

[16] J. Dechargé and D. Gogny, Phys. Rev. C 21, 1568 (1980).

[17] G. F. Bertsch and H. Esbensen, Ann. Phys. (NY) 209, 327 (1991).
[18] E. Garrido, P. Sarriguren, E. Moya de Guerra, and P. Schuck, Phys. Rev. C 60, 064312 (1999).

[19] N. Sandulescu, P. Schuck, and X. Viñas, Phys. Rev. C 71, 054303 (2005).

[20] M. Del Estal, M. Centelles, X. Viñas, and S. K. Patra, Phys. Rev. C 63, 044321 (2001).

[21] M. Kleban, B. Nerlo-Pomorska, J. F. Berger, J. Dechargé, M. Girod, and S. Hilaire, Phys. Rev. C 65, 024309 (2002).

[22] G. Audi, A. H. Wapstra, and C. Thibault, Nucl. Phys. A729, 337 (2003).

[23] J. F. Berger, M. Girod, and D. Gogny, Comput. Phys. Commun. 63, 365 (1991); Nucl. Phys. A502, 85c (1989).

[24] M. Bender, K. Rutz, P.-G. Reinhard, and J. A. Maruhn, Eur. Phys. J. A 8, 59 (2000). 